\title{
DECOMPOSING TENSORS WITH STRUCTURED MATRIX FACTORS REDUCES TO RANK-1 APPROXIMATIONS
}

\author{
Pierre Comon, Mikael Sørensen and Elias Tsigaridas \\ I3S, University of Nice, CNRS \\ Sophia-Antipolis, France
}

\begin{abstract}
Tensor decompositions permit to estimate in a deterministic way the parameters in a multi-linear model. Applications have been already pointed out in antenna array processing and digital communications, among others, and are extremely attractive provided some diversity at the receiver is available. As opposed to the widely used ALS algorithm, non-iterative algorithms are proposed in this paper to compute the required tensor decomposition into a sum of rank-1 terms, when some factor matrices enjoy some structure, such as block-Hankel, triangular, band, etc.
\end{abstract}

\section{MOTIVATION}

This contribution is motivated by the fact that there exist very few efficient numerical algorithms for decomposing a tensor array into a sum of rank- 1 terms. One can just mention the case of symmetric tensors of any order but of dimension 2, which can be decomposed with the help of a Sylvester's theorem [1], or the case of third order tensors having one dimension equal to 2 , which can be handled by computing eigenvectors of a matrix pencil [2]. Even if the case of symmetric complex tensors has been partially solved in [3] , the computational complexity is still significant, since a polynomial system of degree 2 often needs to be solved.

Yet, practical problems are encountered where the factor matrices have a structure [4, 5, 7, 9], such as Toeplitz, which decreases the number of unknowns to be computed. We show in this paper that under certain conditions, the full decomposition can be computed within a finite number of operations (assuming that a matrix SVD can)

The Toeplitz structure has been already exploited in several contributions, e.g. [ 4 , to speed up the ALS algorithm. But the algorithm is still iterative with unproved convergence. Only recently, some authors have attempted to build a noniterative algorithm [5]; however, the latter works in three stages, and can only be applied for a single structured factor. In addition, this factor must be simultaneously Toeplitz lower triangular and banded, which is rather restrictive. The algorithm is suboptimal in the sense that the structure in incompletely exploited, so that it has to be recovered by projection in a third stage.

Tensor decompositions are very attractive in the fields of antenna array processing [6] and digital communications [7],

This work has been partially supported by contract ANR-06-BLAN0074 "Decotes". The work of M. Sørensen is supported by the EU by a Marie-Curie Fellowship (EST-SIGNAL program: http://estsignal.i3s.unice.fr) under contract No MEST-CT-2005-021175. when diversity is available at the receiver. But many other application areas exist [8]. Factor matrices appearing in the tensor decomposition can be structured [4] [9], and can have the very particular structure of banded triangular Toeplitz if Blind Identification of a SISO FIR channel is considered [\$ן A contrario, the algorithms developed in the present paper exploit a structure that can be much less particular, since it is characterized by any linear space of reduced dimension. For instance, only one of the previous features is necessary, e.g. Toeplitz, or triangular, or banded, but not the three of them. We refer to this decomposition as "Structured Canonical Decomposition" (SCAND).

\section{NOTATION}

In order to ease the reading, array symbols are denoted with different fonts, depending on the number of indices. Plain font denotes scalar numbers, e.g. $L, a_{i}$ or $A_{i j}$, boldface lowercases denote vectors, $e . g . \mathbf{x}$, or $\boldsymbol{\alpha}$, boldface uppercases denote matrices, e.g. $\mathbf{A}$, or $\mathbf{S}(\ell)$, and tensor arrays of order higher than 2 are represented by calligraphic letters, e.g. $\mathcal{T}$, or $\mathcal{I}$. In the remainder, $\mathcal{I}$ will always denote the tensor array having ones on its diagonal, and zeros everywhere else.

Tensors are objects defining maps from a product of linear spaces to another. Once the bases of theses spaces are fixed, they are represented by arrays of coordinates. A tensor of order $d$ is represented by an array with $d$ indices. For simplicity, tensors are often (somewhat abusively) assimilated with their array representation; we shall follow this common practice.

Tensor arrays are modified in a multi-linear manner when bases are changed linearly. To make it simple, let $\mathcal{T}$ be a 3 rd order tensor, and let $\mathbf{A}$ (resp. $\mathbf{B}$ and $\mathbf{C}$ ) be linear transforms acting in the first (resp. 2nd and 3rd) linear space. Then the new array representing the tensor can be written as

$$
T_{i j k}^{\prime}=\sum_{\ell m n} A_{i \ell} B_{j m} C_{k n} T_{\ell m n}
$$

which can be conveniently written in a more compact form:

$$
\mathcal{T}^{\prime}=(\mathbf{A}, \mathbf{B}, \mathbf{C}) \cdot \mathcal{T}
$$

This way of denoting a multi-linear transformation is more and more used in the scientific community.

Given two matrices $\mathbf{A}$ and $\mathbf{B}$, one defines the Kronecker product:

$$
\mathbf{A} \otimes \mathbf{B} \stackrel{\text { def }}{=}\left(\begin{array}{ccc}
A_{11} \mathbf{B} & A_{12} \mathbf{B} & \cdots \\
A_{21} \mathbf{B} & A_{22} \mathbf{B} & \cdots \\
\vdots & \vdots &
\end{array}\right)
$$


If the latter matrices have the same number of columns, one also defines the column-wise Kronecker product, often referred to as the Khatri-Rao product:

$$
\mathbf{A} \odot \mathbf{B} \stackrel{\text { def }}{=}[\mathbf{a}(1) \otimes \mathbf{b}(1), \quad \mathbf{a}(2) \otimes \mathbf{b}(2), \quad \cdots] .
$$

Yet another ingredient we shall need is the operation allowing to store a matrix in vector form, $\mathbf{x} \stackrel{\text { def }}{=} \operatorname{vec}\{\mathbf{X}\}$, and the inverse operation, $\mathbf{X}=\operatorname{Unvec}\{\mathbf{x}\}$. To fix the ideas, let $\mathbf{X}$ be a $I \times J$ matrix. We choose the vec $\{\cdot\}$ map defined by $x_{(i-1) J+j}=X_{i j}$. With this definition, we have the property that vec $\left\{\mathbf{x} \mathbf{y}^{\top}\right\}=\mathbf{x} \otimes \mathbf{y}$, for any pairs of vectors $\mathbf{x}$ and $\mathbf{y}$, or equivalently Unvec $\{\mathbf{x} \otimes \mathbf{y}\}=\mathbf{x} \mathbf{y}^{\top}$.

Similarly, tensor arrays can be unfolded into the so-called "unfolding matrices", or "flattening matrices". In the case of order 3 tensors, there are 3 such matrices. Given a tensor $\mathcal{T}$ of dimensions $I \times J \times K$, represented by an arry $T_{i j k}$, the first unfolding matrix is defined as

$$
\mathbf{T}^{(1)}=\left[\begin{array}{c}
\mathbf{T}_{1::} \\
\vdots \\
\mathbf{T}_{I::}
\end{array}\right]
$$

where $\mathbf{T}_{i::}$ denotes the $J \times K$ matrix slice obtained by fixing the 1 st index to $i$ in the tensor array.

Finally, on the linear space of rectangular matrices, one defines the Hermitean scalar product $\langle\mathbf{A}, \mathbf{B}\rangle=\operatorname{trace}\left\{\mathbf{A}^{\mathrm{H}} \mathbf{B}\right\}$; the latter induces the Frobenius norm.

\section{STRUCTURED TENSOR DECOMPOSITION}

We first define the minimal polyadic decomposition of a tensor, which will be referred to as Canonical Decomposition (CAND) The definition is given in the case of a 3rd order tensor, but it extends to any order in an obvious manner. It can be seen as a definition of the tensor rank.

Definition 1 Let $\mathcal{T}$ be a tensor of order 3. $\mathcal{T}$ has rank $R$ if and only if it can be expressed as a sum of $R$ tensor products between 3 vectors, and not fewer than $R$ :

$$
\mathcal{T}=\sum_{p=1}^{R} \mathbf{a}(p) \otimes \mathbf{b}(p) \otimes \mathbf{c}(p)
$$

This holds independently of any basis. Once bases are fixed, the CAND of tensor $\mathcal{T}$ can be equivalently written as a multilinear transform of the diagonal tensor $\mathcal{I}$ :

$$
\mathcal{T}=(\mathbf{A}, \mathbf{B}, \mathbf{C}) \cdot \mathcal{I}
$$

where the factor matrices $\mathbf{A}, \mathbf{B}$ and $\mathbf{C}$ have $R$ columns.

In Definition 1, it is known that factor matrices are not defined in a unique way. In other words, each of them can be post-multiplied by a permutation $\Pi$ and an invertible diagonal matrix, so that $\mathcal{T}=\left(\mathbf{A} \Pi \Lambda_{A}, \mathbf{B} \Pi \Lambda_{B}, \mathbf{C} \Pi \Lambda_{C}\right) \cdot \mathcal{I}$, provided $\boldsymbol{\Lambda}_{A} \boldsymbol{\Lambda}_{B} \boldsymbol{\Lambda}_{C}=\mathbf{I}$. When these indeterminacies generate the whole set of solutions, the decomposition is referred

${ }^{1}$ Note that this decomposition has been named "Parafac" in some communities, i.e. Psychometry. to as essentially unique, and deserves to be called canonical decomposition (CAND) [10]. The lemma below will be useful to choose the permutation and scaling matrices, whenever this indetermination is still present.

Lemma 2 If a matrix $\mathbf{N}$ is invertible, then there exist a permutation $\boldsymbol{\Pi}$ and a diagonal invertible matrix $\boldsymbol{\Lambda}$ such that matrix $\mathrm{N \Pi \Lambda}$ has ones on its diagonal.

Note that if factor matrices are structured, full scaling indeterminacies may disappear, and reduce to a mere scalar scale factor (this is what happens for Toeplitz factors, as addressed subsequently). This is a significant advantage of the SCAND over the CAND. We shall also need the well known results below, that we recall without proof.

Lemma 3 Let $\mathcal{T}$ be a tensor, whose CAND is defined in Def.1. Then its first unfolding matrix can be written as

$$
\mathbf{T}^{(1)}=(\mathbf{A} \odot \mathbf{B}) \mathbf{C}^{\top}
$$

Lemma 4 Denote $\mathbf{T}^{(1)}=\mathbf{U} \mathbf{\Sigma} \mathbf{V}^{\mathrm{H}}$ the SVD of $\mathbf{T}^{(1)}$, where $\boldsymbol{\Sigma}$ is $R \times R$, and $\mathbf{V}^{\mathrm{H}} \mathbf{V}=\mathbf{I}_{R}$. Then, if $\mathbf{A} \odot \mathbf{B}$ and $\mathbf{C}$ are full rank in $(\mathbb{I})$, there always exist $R \times R$ invertible matrices $\mathbf{M}$ and $\mathbf{N}$ such that

$$
\begin{aligned}
& \mathbf{U} \mathbf{\Sigma} \mathbf{M}^{-1}=\mathbf{A} \odot \mathbf{B} \text { and } \mathbf{M} \mathbf{V}^{\mathrm{H}}=\mathbf{C}^{\top} \\
& \mathbf{U N}=\mathbf{A} \odot \mathbf{B} \quad \text { and } \quad \mathbf{N}^{-1} \boldsymbol{\Sigma} \mathbf{V}^{\mathrm{H}}=\mathbf{C}^{\top}
\end{aligned}
$$

If one or several factor matrices appearing in the CAND are imposed to be structured (cf. definition below), we say that we are dealing with a "structured CAND" (SCAND). We shall be concerned by the classes of structured matrices that form linear spaces. Let $\{\mathbf{S}(\ell), 1 \leq \ell \leq I R\}$ be an orthonormal basis of $I \times R$ matrices. We state the following:

Definition 5 An $I \times R$ matrix $\mathbf{S}$ is said to be structured if there exists an orthonormal basis of matrices $\mathbf{S}(\ell)$ such that

$$
\mathbf{S}=\sum_{\ell=1}^{\omega(\mathbf{S})} \alpha(\ell) \mathbf{S}(\ell)
$$

where $\omega(\mathbf{S})<I R$ is given. Such linear spaces will be denoted $\mathcal{A}, \mathcal{B}, \mathcal{C}$ for factor matrices $\mathbf{A}, \mathbf{B}$ and $\mathbf{C}$, respectively.

For instance, strictly lower triangular matrices are structured in the above sense, as well as Toeplitz or Hankel matrices, skew-symmetric matrices, and certain band matrices. Assume only matrix $\mathbf{C}$ is structured in (1); then we have:

Lemma 6 If the linear space $\mathcal{C}$ is stable by postmultiplication by invertible diagonal matrices, then matrix $\mathbf{M}$ defined in Lemma $¥$ can be imposed to have ones on its diagonal. Otherwise, one can always impose $M_{r 1}=1$, or $\gamma(r)=1$ for some $r$.

Proof. From equation (2), it is clear that pre-multiplication of $\mathbf{C}^{\top}$ by $\Lambda \Pi^{\top}$ implies pre-multiplication of $\mathbf{M}$ by the same factor. Then by Lemma 2 , one can choose scaling matrix $\Lambda$ and permutation $\Pi$ so that $\operatorname{Diag}\{\mathbf{M}\}=\mathbf{I}$. Now when $\mathbf{C} \Pi \boldsymbol{\Lambda}$ does not always belong to $\mathcal{C}$, such a choice is not possible. But a scalar scale factor always subsists in (1), such that any non-zero entry of $\mathbf{M}$ can be set to 1 , and there is at 
least one in the 1 st column. Another choice consists of using this scale factor to impose a nonzero parameter in the right hand side of (4) to be equal to 1, e.g. $\gamma(1)=1$.

With a similar reasoning one can state the following

Lemma 7 If both $\mathbf{A}$ and $\mathbf{B}$ are structured in (17), but not $\mathbf{C}$, one can always impose either $M_{11}=1$, or $\alpha(1)=1=\beta(1)$.

\section{NON-ITERATIVE SOLUTIONS}

In this section, results allowing to deflate the CAND to matrix SVD's will be stated.

Proposition 8 Let $\mathcal{T}$ be a tensor of dimensions $I \times J \times K$ and rank $R>1$, admitting the SCAND below:

$$
\mathcal{T}=(\mathbf{A}, \mathbf{B}, \mathbf{C}) \cdot \mathcal{I}
$$

where matrix $\mathbf{C}$ is structured (according to Def. 5) with $\omega(\mathbf{C}) \leq K^{2} / 4$. Then, the calculation of the three matrix factors may be achieved by solving a linear system followed by $R$ matrix rank-1 approximations, provided the rank of $\mathcal{T}$ is not too large, namely

$$
R^{2}-K R+\omega(\mathbf{C})-1 \leq 0
$$

Proof. Since $\mathbf{C}$ is structured, it can be written as $\mathbf{C}=$ $\sum_{\ell=1}^{\omega(\mathbf{C})} \gamma(\ell) \mathbf{C}(\ell)$ where matrices $\mathbf{C}(\ell)$ are known. Since $\mathcal{T}$ is of rank $R>1, \mathbf{C}$ is nonzero, and from Lemma 6 , we may set $\gamma(1)=1$. Then from Lemma 4 , matrix $\mathbf{M}$ must satisfy:

$$
\mathbf{M} \mathbf{V}^{\mathrm{H}}=\mathbf{C}(1)+\sum_{\ell=2}^{\omega(\mathbf{C})} \gamma(\ell) \mathbf{C}(\ell)^{\top}
$$

This linear system contains $K R$ equations and $R^{2}+\omega(\mathbf{C})-1$ unknowns, $\gamma(k)$ and $M_{i j}$. Since it has more equations than unknowns, according to our assumption, it generically admits one solution. We have thus obtained matrices $\mathbf{M}$, and $\mathbf{C}$.

On the other hand, we have from (2) that $\mathbf{F} \stackrel{\text { def }}{=}$ $\mathbf{U} \boldsymbol{\Sigma} \mathbf{M}^{-1}=\mathbf{A} \odot \mathbf{B}$. The last operation remaining to perform is the calculation of matrices $\mathbf{A}$ and $\mathbf{B}$, which can be done in a standard way column by column. For doing this, one notices that the $r$ th column of matrix $\mathbf{F}, \mathbf{f}(r)$, is ideally equal to $\mathbf{a}(r) \otimes \mathbf{b}(r)$, whose matrix unvectorization form is $\mathbf{a}(r) \mathbf{b}(r)^{\top}$. So estimates of columns $\mathbf{a}(r)$ and $\mathbf{b}(r)$ of $\mathbf{A}$ and $\mathbf{B}$ can indeed be obtained by computing the best rank-1 approximate of matrix Unvec $\{\mathbf{f}(r)\}$.

Proposition 9 Let $\mathcal{T}$ be a tensor of dimensions $I \times J \times K$ and rank $R$, admitting the $\mathrm{CAND}: \mathcal{T}=(\mathbf{A}, \mathbf{B}, \mathbf{C}) \cdot \mathcal{I}$, where matrices $\mathbf{A}$ and $\mathbf{B}$ are structured, possibly with different structures, with $\omega(\mathbf{A}) \omega(\mathbf{B}) \leq I^{2} J^{2} / 4$. Then, the calculation of the three matrix factors can be achieved by solving a linear system followed by one matrix rank-1 approximation, provided the rank of $\mathcal{T}$ satisfies the necessary condition:

$$
R^{2}-I J R+\omega(\mathbf{A}) \omega(\mathbf{B})-1 \leq 0 .
$$

Proof. Since matrices A and $\mathbf{B}$ are structured, we have

$$
\mathbf{U} \mathbf{N}=\sum_{i=1}^{\omega(\mathbf{A})} \sum_{j=1}^{\omega(\mathbf{B})} \alpha(i) \beta(j)(\mathbf{A}(i) \odot \mathbf{B}(j))
$$

which contains $I J R$ equations, using the notation of (3). System (8) can be seen as a linear system of $I J R$ equations in the $\omega(\mathbf{A}) \omega(\mathbf{B})$ unknowns $X(i, j) \stackrel{\text { def }}{=} \alpha_{i} \beta_{j}$. Next, from Lemma 7, if we choose to impose $N_{11}=1$, we also have $R^{2}-1$ unknowns $N_{i j}$. Note that if spaces $\mathcal{A}$ and $\mathcal{B}$ are stable by diagonal scaling, we impose instead $\operatorname{Diag}\{\mathbf{N}\}=\mathbf{I}$, and we have $R^{2}-R$ remaining unknowns; but let's concentrate on the less favorable case $N_{11}=1$.

Hence, linear system (8) contains $I J R$ equations in $R^{2}-$ $1+\omega(\mathbf{A}) \omega(\mathbf{B})$ unknowns. It generically suffices to determine matrices $\mathbf{N}$ and consequently $\mathbf{C}$, as well as matrix $\mathbf{X}$, because $R$ is not too large, by hypothesis.

The last step consists of computing the best rank-1 approximate of matrix $\mathbf{X}, \boldsymbol{\alpha} \boldsymbol{\beta}^{\top}$, as in the proof of Proposition 8, which will yield $\mathbf{A}$ and $\mathbf{B}$.

Now if all three factor matrices are structured, one can show that their estimation can be carried out with the help of a rank-1 approximate of a 3rd order tensor, which today still needs an iterative algorithm. The procedure is described in the next section.

There is however yet another case where the CAND can be computed by solely resorting to matrix SVD's, as shown in the proposition below.

Proposition 10 Let $\mathcal{T}$ be a tensor of order 4, with 2 structured matrix factors. Then under the same conditions as in Prop. 9, its SCAND may be computed by solving an overdetermined linear system, and by computing $R+1$ rank-1 matrix approximates.

Proof. Consider a $I \times J \times K \times L$ tensor, and its $I J \times K L$ unfolding matrix:

$$
\mathbf{T}^{(2,2)}=(\mathbf{A} \odot \mathbf{B})(\mathbf{C} \odot \mathbf{D})^{\top}
$$

where $\mathbf{A}, \mathbf{B}, \mathbf{C}, \mathbf{D}$ all have $R$ columns. We assume that both $\mathbf{A}$ and $\mathbf{B}$ are structured, which means that we have

$$
\mathbf{T}^{(2,2)}=\sum_{i=1}^{\omega(\mathbf{A})} \sum_{j=1}^{\omega(\mathbf{B})} \alpha_{i} \beta_{j}(\mathbf{A}(i) \odot \mathbf{B}(j))(\mathbf{C} \odot \mathbf{D})^{\top}
$$

As in the proof of Proposition 9, we consider the SVD of matrix $\mathbf{T}^{(2,2)}=\mathbf{U} \boldsymbol{\Sigma} \mathbf{V}^{\mathrm{H}}$. Hence there exists a $R \times R$ invertible matrix, $\mathbf{N}$, such that $\mathbf{U} \mathbf{N}=\mathbf{A} \odot \mathbf{B}$ and $\mathbf{N}^{-1} \boldsymbol{\Sigma} \mathbf{V}^{\mathrm{H}}=$ $\mathbf{C} \odot \mathbf{D}$. Our linear system contains $I J R$ equations and $R^{2}-1+\omega(\mathbf{A}) \omega(\mathbf{B})$ unknowns, $N_{i j}$ and $X_{p q}$, if we set $N_{11}=1$. Once this over-determined system has been solved in the Least Squares (LS) sense, matrices $\mathbf{N}$ and $\mathbf{X}$ are known. Next we obtain again $\alpha_{i}$ and $\beta_{j}$ via a rank-one approximation of matrix $\mathbf{X}$.

It remains to solve $\mathbf{N}^{-1} \boldsymbol{\Sigma} \mathbf{V}^{\mathrm{H}}=(\mathbf{C} \odot \mathbf{D})^{\top}$. Following the same lines as in the proof of Proposition 8, denote $\mathbf{F}=\mathbf{V}^{*} \boldsymbol{\Sigma} \mathbf{N}^{-\mathrm{T}}$ and $\mathbf{f}(r)$ its columns, $1 \leq r \leq R$, each of dimension $K L$. The rest of the proof is similar to that of Proposition 8, viz, the columns $\mathbf{a}(r)$ and $\mathbf{b}(r)$ are obtained by computing the best rank-1 approximation of the $K \times L$ matrices Unvec $\{\mathbf{f}(r)\}$, respectively. 


\section{SOLUTIONS REQUIRING HIGHER ORDER RANK-1 APPROXIMATES}

From the proofs derived in the previous section, it is clear that our propositions can be extended to tensors having three structured matrices, or to tensors of order larger than 3 . One such instance is given below; the proof is not reproduced here for reasons of space.

Proposition 11 Let $\mathcal{T}$ be a tensor of order d and dimensions $K_{\mu}, 1 \leq \mu \leq d$, with $m$ structured matrix factors, $m>0$, and $d-m$ unstructured. Then its SCAND can be computed by solving an overdetermined linear system, and by computing rank-1 approximations of one tensor of order $m$ and $R$ tensors of order $d-m$, provided conditions below are met:

$$
\begin{array}{r}
\prod_{\mu=1}^{m} \omega\left(\mathbf{A}^{(\mu)}\right)-\frac{1}{4} \prod K_{\mu}^{2} \leq 0 \\
R^{2}-\left(\prod_{\mu=1}^{m} K_{\mu}\right) R+\prod_{\mu=1}^{m} \omega\left(\mathbf{A}^{(\mu)}\right)-1 \leq 0
\end{array}
$$

\section{EXAMPLES}

\subsection{One banded Toeplitz factor}

Assume the $K \times R$ matrix factor $\mathbf{C}$ is Toeplitz lower triangular with bandwidth $\omega(\mathbf{C})=K-R+1$. If the first matrix $\mathbf{C}(1)$ in the basis is the identity, assuming $\gamma(1)=1$ means that we can assume $\mathbf{C}$ has ones on its diagonal. Beside the identity matrix $\mathbf{I}$, the next basis matrices $C(\ell)$ have ones on their $\ell$ th subdiagonal, and are null elsewhere: $C(\ell)_{i j}=\delta(i-j-$ $\ell), i>j$, where $\delta(\cdot)$ denotes the Kronecker delta. In other words:

$$
\mathbf{C}=\mathbf{I}+\sum_{\ell=2}^{\omega(\mathbf{C})} \gamma(\ell) \mathbf{C}(\ell)
$$

The remaining $K R-\omega(\mathbf{C})-1$ basis matrices may be obtained in a non unique manner by completion, under the orthonormality constraint. The rank condition (5) becomes, as a function of $\omega(\mathbf{C})$ :

$$
\omega(\mathbf{C})^{2}-(K+1) \omega(\mathbf{C})+2 K \leq 0
$$

It can be checked that this condition admits solutions only for $K>5$. For instance, for $K=20$, the above condition becomes $2<\omega(\mathbf{C})<19$.

\subsection{One Hankel factor}

Assume the $K \times R$ matrix factor $\mathbf{C}$ is Hankel. It is characterized by $\omega(\mathbf{C})=K+R-1$ free parameters. The $\ell$ th basis matrix $1 \leq \ell \leq \omega(\mathbf{C})$ is Hankel with a single nonzero antidiagonal, that is: $C(\ell)_{i j}=\delta(i+j-\ell-1)$.

\subsection{Two banded Toeplitz factors}

We considered next a more general banded Toeplitz case. We took two factors with the same structure, i.e. $I=J$ and $\omega(\mathbf{A})=\omega(\mathbf{B})=\omega$. The rank condition (7) can be expressed as a function of $\omega$ as $R^{2}-I^{2} R+\omega^{2}-1 \leq 0$. Simulations have
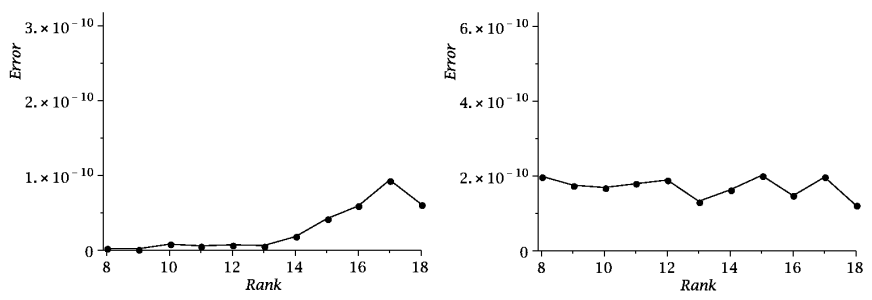

Fig. 1. Relative reconstruction error for a $20 \times 20 \times 20$ tensor. Left: one Toeplitz factor; Right: two Toeplitz factors.

been run for $I=20,8 \leq R \leq 19$, and $\omega=18$, with 12 subdiagonals and 5 superdiagonals. The computer experiments reported in Fig. 1 have been executed under these conditions, with one or two structured matrices, the other factors being drawn randomly. See [11] for further (e.g. noisy) results.

\section{CONCLUDING REMARKS}

Several other computer results could not be reported for reasons of space; see [11]. When two matrices are structured, their identification conditions are easier to meet than in the case when only one is structured. Maple and Matlab computer codes will be made available after publication.

\section{REFERENCES}

[1] P. Comon and B. Mourrain, "Decomposition of quantics in sums of powers of linear forms," Signal Processing, Elsevier, vol. 53, no. 2, pp. 93-107, Sept. 1996, special issue.

[2] J. M. F. Ten Berge, "The typical rank of tall three-way arrays," Psychometrika, vol. 65, no. 5, pp. 525-532, Sept. 2000.

[3] J. Brachat, P. Comon, B. Mourrain, and E. Tsigaridas, "Symmetric tensor decomposition," Linear Algebra and its Applications, 2009, submitted, arXiv:0901.3706.

[4] D. Nion and L. De Lathauwer, "A block component model based blind DS-CDMA receiver," IEEE Trans. Signal Proc., vol. 56, no. 11, pp. 5567-5579, 2008.

[5] A. Kibangou and G. Favier, "Non iterative solution for Parafac with a Toeplitz factor," in Eusipco'09, Glasgow, UK, Aug. 2428 2009, Eurasip.

[6] N. D. Sidiropoulos, R. Bro, and G. B. Giannakis, "Parallel factor analysis in sensor array processing," IEEE Trans. Sig. Proc., vol. 48, no. 8, pp. 2377-2388, Aug. 2000.

[7] N. D. Sidiropoulos, G. B. Giannakis, and R. Bro, "Blind PARAFAC receivers for DS-CDMA systems," IEEE Trans. on Sig. Proc., vol. 48, no. 3, pp. 810-823, Mar. 2000.

[8] A. Smilde, R. Bro, and P. Geladi, Multi-Way Analysis, Wiley, 2004.

[9] A. L. F. de Almeida, G. Favier, and J. C. M. Mota, "Parafacbased unified tensor modeling for wireless communication systems with application to blind multiuser equalization," Signal Processing, vol. 87, no. 2, pp. 337-351, Feb. 2007.

[10] P. Comon, X. Luciani and A. L. F. de Almeida, "Tensor Decompositions, Alternating Least Squares and other Tales," $J$. Chemometrics, 23:393-405, Aug. 2009.

[11] P. Comon, M. Sørensen and E. Tsigaridas, Research report I3S/RR-2009-11, Sept. 8, 2009. 